This is an author-created, un-copyedited version of an article accepted for publication/published in Modelling and Simulation in Materials Science and Engineering. IOP Publishing Ltd is not responsible for any errors or omissions in this version of the manuscript or any version derived from it. The Version of Record is available online at http://dx.doi.org/10.1088/0965-0393/23/8/085008. Citation details: Leyson, G. P. M.; Curtin, W. A. Model. Simul. Mater. Sci. Eng. 2016, 24 (6), 65005.

\title{
Robust Atomistic Calculation of Dislocation Line Tension
}

\author{
B A Szajewski ${ }^{1,2}$, F Pavia ${ }^{2}$, W A Curtin ${ }^{2}$ \\ ${ }^{1}$ School of Engineering, Brown University, Providence, RI, 02912 \\ ${ }^{2}$ Institute of Mechanical Engineering, EPFL, Lausanne, Switzerland 1015 \\ E-mail: Benjamin_Szajewski@brown.edu
}

\begin{abstract}
The line tension $\Gamma$ of a dislocation is an important and fundamental property ubiquitous to continuum scale models of metal plasticity. However, the precise value of $\Gamma$ in a given material has proven difficult to assess, with literature values encompassing a wide range. Here results from a multiscale simulation and robust analysis of the dislocation line tension, for dislocation bow-out between pinning points, are presented for two widely-used interatomic potentials for Al. A central part of the analysis involves an effective Peierls stress applicable to curved dislocation structures that markedly differs from that of perfectly straight dislocations but is required to describe the bow-out both in loading and unloading. The line tensions for the two interatomic potentials are similar and provide robust numerical values for Al. Most importantly, the atomic results show notable differences with singular anisotropic elastic dislocation theory in that (i) the coefficient of the $\ln (L)$ scaling with dislocation length $L$ differs and (ii) the ratio of screw to edge line tension is smaller than predicted by anisotropic elasticity. These differences are attributed to local dislocation core interactions that remain beyond the scope of elasticity theory. The many differing literature values for $\Gamma$ are attributed to various approximations and inaccuracies in previous approaches. The results here indicate that continuum line dislocation models, based on elasticity theory and various core-cut-off assumptions, may be fundamentally unable to reproduce full atomistic results, thus hampering the detailed predictive ability of such continuum models.
\end{abstract}

Keywords: Dislocation Line Tension, Multiscale, Atomistic Simulation. 
This is an author-created, un-copyedited version of an article accepted for publication/published in Modelling and Simulation in Materials Science and Engineering. IOP Publishing Ltd is not responsible for any errors or omissions in this version of the manuscript or any version derived from it. The Version of Record is available online at http://dx.doi.org/10.1088/0965-0393/23/8/085008. Citation details: Leyson, G. P. M.; Curtin, W. A. Model. Simul. Mater. Sci. Eng. 2016, 24 (6), 65005.

Robust Atomistic Calculation of Dislocation Line Tension

\section{Introduction}

The onset of flow in metals is governed by the inhibition of dislocation glide through the crystalline lattice. Dislocations are pinned by various types of obstacles, with applied stress and thermal activation allowing the dislocations to move through a field of obstacles [1-16]. Increasingly sophisticated models are guiding the understanding of these processes, and their consequences on observable/measurable phenomena [17-21]. Central to many of these models is the concept of the dislocation line tension $\Gamma$, which is associated with the energy required to form curved dislocation structures. Formally, the line tension is related to the infinitesimal change in energy $\delta W$ associated with an infinitesimal change in dislocation length $\delta S[22-25]$

$$
\Gamma=\lim _{S \rightarrow 0} \frac{\delta W}{\delta S} .
$$

Dislocation line tension is thus a function of the dislocation configuration, which is implicitly contained in $W$ and $S$. For simplicity, the line tension $\Gamma$ is often assumed to be a local quantity, i.e. associated with local line length and curvature. However, $\Gamma$ actually consists of contributions from both the local increase in dislocation length and the longer-range interactions among all segments of the dislocation line, which leads to a $\ln (L)$ scaling with line length $L$. A simple approximation, $\Gamma \approx \frac{1}{2} \mu b^{2}$ [10,11,26-32], where $\mu$ is the shear modulus and $b$ the Burgers vector was originally proposed over half a century ago [30,31]. A more nuanced model accounts for the long-range interactions by modifying the prefactor of $1 / 2$ and adding the $\ln (L)$ scaling that emerges from elasticity. Such estimates of $\Gamma$ have provided insight into a range of key processes in materials, such as dislocation junction formation and breaking, [27, 33-35], dislocation-obstacle interactions $[1,5,8,9]$, and solute strengthening models [20,21]. Many of these models for dislocation pinning by obstacles lead to predictions for the critical resolved shear stress (CRSS) $\tau_{c}$ of the form

$$
\tau_{c}=\alpha \Gamma / b L
$$

where $L$ is the distance between pinning points, and $\alpha$ is a parameter related to the local dislocation/obstacle interactions. Additionally, numerical methods (2D [36] and some 3D discrete dislocation dynamics [37-40]) rely on a phenomenological line tension to avoid the singular fields associated with elasticity. Continuum dislocation line models also rely on the assumption that dislocations are adequately represented by smooth curves or discretized straight segments, and that the atomic-scale phenomena of the Peierls stress $\tau_{P}$ can be accurately represented as a homogeneous stress opposing motion of the dislocation. If these assumptions are valid, direct atomistic simulations are then also required to calibrate the "material parameters" (line tension $\Gamma$, dislocation core energy $E_{\text {core }}$, Peierls stress $\tau_{P}$, dislocation mobility $B$, and their variations with dislocation character ranging from screw to edge) that enter into the continuum model. As computational materials science moves into an era of increasing accuracy and predictive capability for $\tau_{c}$, accurate values for all of the material parameters, including dislocation line tension, become increasingly important. 
This is an author-created, un-copyedited version of an article accepted for publication/published in Modelling and Simulation in Materials Science and Engineering. IOP Publishing Ltd is not responsible for any errors or omissions in this version of the manuscript or any version derived from it. The Version of Record is available online at http://dx.doi.org/10.1088/0965-0393/23/8/085008. Citation details: Leyson, G. P. M.; Curtin, W. A. Model. Simul. Mater. Sci. Eng. 2016, 24 (6), 65005.

As a result of this importance, and with the increasing availability of efficient parallelized molecular dynamics algorithms [41], a number of attempts have been made to calculate the line tension directly from atomistic simulations [2, 20, 42-48]. The simplest realization of such a calculation is the periodic small bow-out problem [22] in which an infinite initially-straight dislocation is pinned at a periodic array of obstacles with a spacing $L$. A driving resolved shear stress $\tau_{a p p}$ is then applied, causing the free regions of the dislocation to bow-out in between the pinning points, as shown in figure 1; this problem is thus closely related to models of strengthening via pinning of dislocations. The work $\tau_{a p p} b d \mathrm{~A}$ done by $\tau_{a p p}$ over the incremental area $d \mathrm{~A}$, over which the upper and lower surfaces are displaced relatively by one Burgers vector $b$, is balanced by the additional energy $\Gamma d S$ of the line tension eq. 1 associated with the incremental increased length $d S$ of the dislocation. An equilibrium configuration is achieved when these incremental energies (i.e. configurational forces) are equal, so that the line tension can be directly related to the evolving shape of the dislocation as

$$
\Gamma=\tau_{a p p, e f f} b \frac{\mathrm{d} A}{\mathrm{~d} S}
$$

Bacon, Osetsky and Rodney [49] have reviewed many of the methods employed in this type of calculation for obstacles such as clusters of solute atoms, vacancies, and recently forest dislocations. Unfortunately, previous atomistic studies have yielded vastly different results for the same dislocation in the same material, e.g. an edge dislocation in $\mathrm{Al}$ as described by a given interatomic potential. Attempts have been made to identify and compensate for spurious boundary-induced stresses $[44,50,51]$, inertial effects [42], artificial pinning-point interactions [2,6], and the Peierls stress $\tau_{P}$. The Peierls stress, usually measured on a straight dislocation, is either treated as a uniform applied internal back stress so that the effective applied stress is $\tau_{a p p}-\tau_{P}$ or is neglected. After such adjustments/corrections, however, the literature values for the line tension of an edge dislocation in Al using the Ercolessi-Adams (EA) EAM potential range from $0.06 \leq \Gamma \leq 1 \mathrm{eV} / \AA[2,20,42,44]$. Since the CRSS $\tau_{c}$ is directly proportional to $\Gamma$, per eq. 2 above, this range of estimates suggests an order-of-magnitude uncertainty in the CRSS in a given material system, which is obviously unacceptable for guiding the design of new materials.

In this paper, we report a robust and accurate atomic-level measurement of the line tension for both edge and screw dislocations in $\mathrm{Al}$ within the context of the small bowout problem described above (see figure 1). We use an accurate 3D multiscale method that is devoid of spurious image forces common in finite-size MD simulations [52], and use eq. 3 directly to measure the line tension. We also describe carefully how an effective Peierls stress $\tau_{P}$ enters into the analysis so as to yield consistent results for both loading and unloading of bowed dislocations, and discuss the conditions over which consistent results can be obtained. Precise values for the line tension are then obtained that are weakly dependent on the interatomic potential. We obtain a $\ln (L)$ scaling but with a coefficient that differs somewhat from the prediction of anisotropic elasticity, and we find a ratio of $\Gamma_{\text {screw }} / \Gamma_{\text {edge }}$ that differs significantly from anisotropic elasticity predictions; 
This is an author-created, un-copyedited version of an article accepted for publication/published in Modelling and Simulation in Materials Science and Engineering. IOP Publishing Ltd is not responsible for any errors or omissions in this version of the manuscript or any version derived from it. The Version of Record is available online at http://dx.doi.org/10.1088/0965-0393/23/8/085008. Citation details: Leyson, G. P. M.; Curtin, W. A. Model. Simul. Mater. Sci. Eng. 2016, 24 (6), 65005.

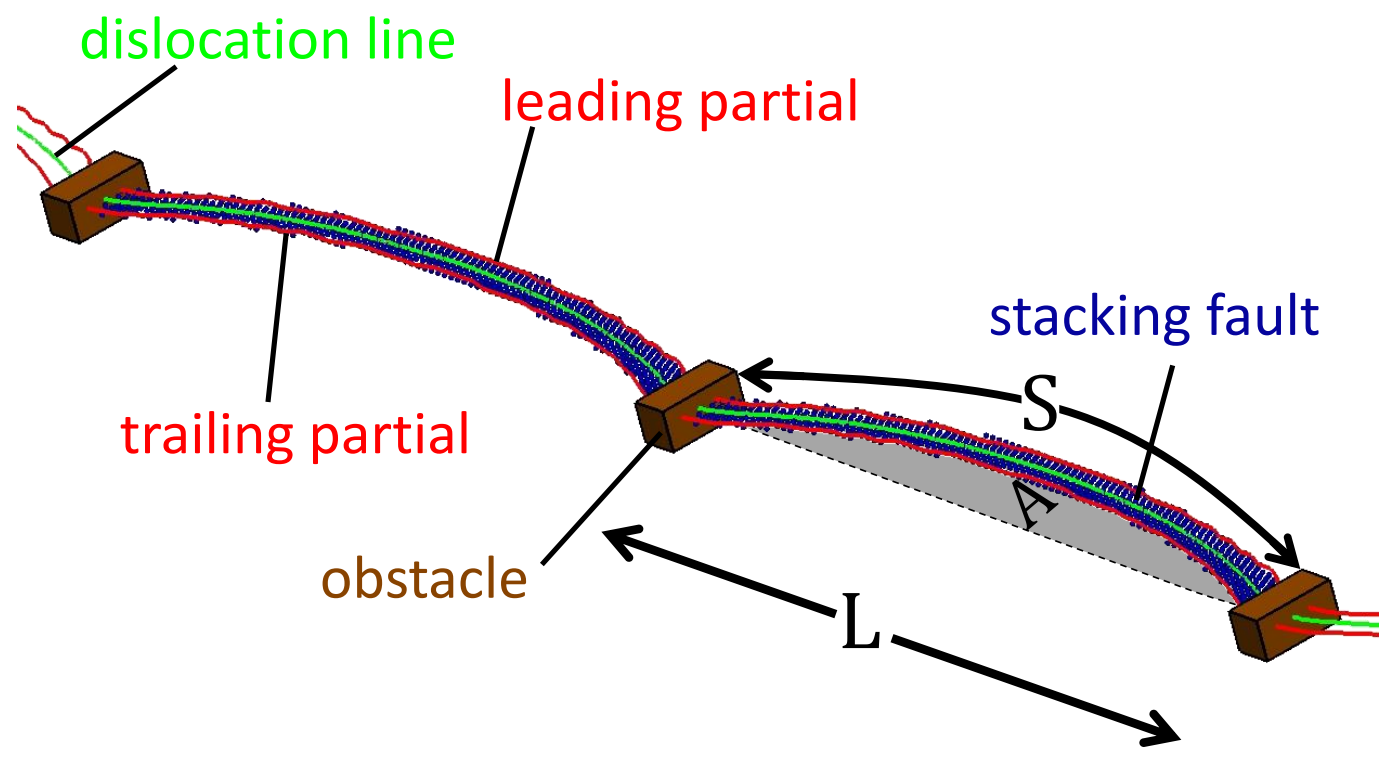

Figure 1. Schematic of the dislocation-obstacle bow-out configuration. The simulation cell is periodic along the line direction with length $L$ and pinned by a periodic array of obstacles. Under a homogeneous shear stress or strain, the dislocation bows out into some equilibrium shape having contour length $S$ and swept-out area $A$. In fcc materials, the full dislocation dissociates into two Shockely partial dislocations (red) with a stacking fault (blue) in between; the averaged smoothed dislocation line (green) determines $S$ and $A$ relative to the initial straight dislocation.

these differences are attributed to fundamental features of curved atomistic dislocations that are absent from continuum elasticity theory.

The remainder of this paper is organized as follows. In Sec. 2, we review the elastic theory for line tension to set a clear background for our analysis. In Sec. 3, we describe our simulation and analysis methods. In Sec. 4, we present and discuss our results in depth. In Sec. 5, we discuss important implications of our results for both materials science applications and continuum discrete-dislocation simulations, and then summarize our major results.

\section{Review of Line Tension within Isotropic and Anisotropic Elasticity}

The line tension has been widely studied within the elastic theory of dislocations for both isotropic [22,32] and anisotropic [53-55] materials. Within elasticity theory, the Burgers vector displacement discontinuity is assumed to terminate abruptly at the dislocation core resulting in a local, weak divergence in the elastic energy. Analytical and numerical results for the self- and interaction-energies of discrete dislocation segments thus require the introduction of ad-hoc core-cut-offs or regularization procedures. Denoting the (singular) dislocation self-interaction cut-off radius as $r_{0}$, the elastic energy of a discrete dislocation segment may be denoted as $W_{\text {self }}\left(r_{0}\right)$ [22], and no additional core energy 
This is an author-created, un-copyedited version of an article accepted for publication/published in Modelling and Simulation in Materials Science and Engineering. IOP Publishing Ltd is not responsible for any errors or omissions in this version of the manuscript or any version derived from it. The Version of Record is available online at http://dx.doi.org/10.1088/0965-0393/23/8/085008. Citation details: Leyson, G. P. M.; Curtin, W. A. Model. Simul. Mater. Sci. Eng. 2016, 24 (6), 65005.

is added. Cai and coworkers [56] proposed an isotropic elastic non-singular theory that smears the core over a finite region of length $r_{c}$ to eliminate the singularity and included an additional core energy $E_{\text {core }}$, resulting in a discrete dislocation segment energy $W_{\text {self }}\left(r_{c}, E_{\text {core }}\right)$ [57]. In both theories, the total energy of an arbitrary dislocation configuration is computed by summing the self and interaction energies of each discrete segment $\left(W=\sum W_{\text {self }}+\sum W_{\text {int }}\right)$, from which forces can then be derived and the dislocation configuration evolved in time; this is achieved within various available 3DDiscrete Dislocation codes such as ParaDis [37].

Within the above theories, the line tension eq. 1 can be computed for various problems such as the small periodic bow-out problem of figure 1. Usually, for a given parametric geometry (triangle, sinusoid, arc of a circle, and periodic or not), the total energies $W(S)$ and $W(S+\delta S)$ are computed for two self-similar geometries having an incremental increase in bow-out length from $S$ to $S+\delta S$. Following eq. 1 the line tension is the (energetic) difference between these two configurations and is computed as $\Gamma^{e l}=[W(S+\delta S)-W(S)] / \delta S$ and in the limit $\delta S \rightarrow 0$ we recover eq. 1. Following Hirth and Lothe $(1982,[22])$, the line tension $\Gamma^{e l}$ resulting from such calculations can be written as a term linear in $\ln (L)$ plus a constant which, including a general $E_{\text {core }}$ term, yields

$$
\Gamma^{e l}=C_{1}(\phi) \ln (L / b)+C_{0}\left(\phi ; \frac{r_{0}}{b}, E_{\text {core }}\right)
$$

where $\phi$ is the character angle of the initial straight dislocation and small bow-out is assumed. Both incremental geometries corresponding to $S$ and $S+\delta S$ are thus incremental with respect to the original straight dislocation. In isotropic elasticity theory, the first term of the above equation is

$$
\begin{aligned}
C_{1}(\phi) \ln (L / b) & =\left(W_{\text {self }}(\phi)+\frac{\partial^{2} W_{\text {self }}(\phi)}{\partial \phi^{2}}\right) \\
& =\left(\frac{\mu b^{2}}{4 \pi(1-\nu)}\right)\left(1+\nu\left[\cos ^{2}(\phi)-2 \sin ^{2}(\phi)\right]\right) \ln (L / b)
\end{aligned}
$$

The $C_{1}$ prefactor is thus well defined. The term $C_{0}$ depends on the incremental change in interaction energies $\left(\sum \delta W_{\text {int }} / \delta S\right)$, which are geometry specific, and also the arbitrary (singular) cut-off parameter $r_{0} / b$ and the core energy $E_{\text {core }}$, and can be written as [57],

$$
C_{0}\left(\phi ; \frac{r_{0}}{b}, E_{\text {core }}\right)=-C_{1}(\phi) \ln \left(r_{0} / b\right)+C_{1}(\phi)\left(4 \pi E_{\text {core }} / \mu\right)+C_{00}(\phi)
$$

Table 1 shows the computed results for the $\ln (L / b)$ prefactor $\mathrm{C}_{1}$ and the constant $\mathrm{C}_{0}$ for several similar bow-out geometries, using (singular) cut-off parameter $r_{0} / b=1$ and $E_{\text {core }}=0$ so that the quoted value coincides with $C_{00}$, for initially straight screw $(\phi=0)$ and edge $(\phi=90)$ dislocations. For specific numerical computations, we use the isotropic constants of the Ercolessi-Adams Al [59] interatomic potential ( $\mu=30.8 \mathrm{e} 9, \nu=0.35)$ with Burgers vector $\mathrm{b}=2.851 \AA$. While $E_{\text {core }}$ itself, as implemented within ParaDiS, has no character dependence the term appearing within the line tension, $\left(C_{1}(\phi) \times E_{\text {core }}\right)$ does 
This is an author-created, un-copyedited version of an article accepted for publication/published in Modelling and Simulation in Materials Science and Engineering. IOP Publishing Ltd is not responsible for any errors or omissions in this version of the manuscript or any version derived from it. The Version of Record is available online at http://dx.doi.org/10.1088/0965-0393/23/8/085008. Citation details: Leyson, G. P. M.; Curtin, W. A. Model. Simul. Mater. Sci. Eng. 2016, 24 (6), 65005.

Robust Atomistic Calculation of Dislocation Line Tension

\begin{tabular}{lcc}
\hline Type & $\mathrm{C}_{1} / \frac{\mu b^{2}}{4 \pi(1-\nu)}$ & $\mathrm{C}_{0} / \frac{\mu b^{2}}{4 \pi(1-\nu)}$ \\
\hline Small Triangular Bow-out & & \\
Edge [22] & 0.33 & -1.31 \\
Screw [22] & 1.33 & -3.51 \\
Small-amplitude Sinusoid & & \\
Edge & 0.33 & -1.32 \\
Screw [58] & 1.33 & -3.54 \\
Periodic Small Bow-out & & \\
Elasticity & 0.33 & -1.49 \\
Edge & 1.33 & -4.20 \\
Screw & & \\
Periodic Small Bow-out (NS Theory [56]) & 0.33 & -0.92 \\
Edge & 1.20 & -4.15 \\
Screw & & \\
\hline
\end{tabular}

Table 1. Line Tension $\left(\Gamma^{e l}\right)$ coefficients for several characteristic bow-out problems $\nu$ is taken to be $1 / 3$, the singular cut-off parameter $r_{0}=|\mathbf{b}|$, the non-singular cut-off parameter $\mathrm{r}_{c}=|\mathbf{b}| e$ and $E_{\text {core }}=0$.

(see $[57,60]$ for further details). Also shown are the analytic results for the non-periodic small triangle bow-out of [22] and the small-amplitude sinusoidal bow-out [58], and also the computed results for NS theory with $r_{c} / b=e$ replacing $r_{0} / b=1$. Aside from a small deviation for the NS screw case, the $C_{1}$ coefficient is the same in all of these problems and the ratio of screw to edge values is $\approx 4$, as is well-known. The coefficient $C_{00}$ differs slightly with the geometry and theory, because of the chosen cut-off and core parameters, but all cases give fairly similar results. Any additional explicit core energy can be added. Thus, elasticity theory provides a robust value for the coefficient $C_{1}$ associated with the $\ln (L / b)$ scaling but, in the absence of atomistic information about the core energy, does not provide any absolute line energy or line tension.

The elastic term $C_{1} \ln (L / b)$ dominates for sufficiently large $L$, and so the $C_{0}$ term and core energy are often neglected. However, with no core energy, elasticity predicts line tension to be negative at "small" $L$. Since $C_{0} \approx 4 C_{1}$, the line tension is zero at $\ln (L / b) \sim 4$ or $L \approx 16 \mathrm{~nm}$ for $b \approx 0.3 \mathrm{~nm}$. Dislocation densities relevant in real materials range from from $10^{12}-10^{15} / \mathrm{m}^{2}$, so that the typical spacing between dislocation junctions is $L \sim 30-300 \mathrm{~nm}$, a range also comparable to precipitate spacings in many alloys. The core energy is therefore essential at the lowest relevant scales, $\ln (L / b) \approx 4$ and is likely non-negligible up to $\ln (L / b) \sim 6$ corresponding to $L \approx 120 \mathrm{~nm}$. An accurate core energy and elimination of arbitrary cut-off parameters are thus necessary to obtain a line tension value that would be accurate for many materials science applications, assuming that elasticity is otherwise valid.

Within anisotropic elasticity theory, the line energy and line tension depend not 
This is an author-created, un-copyedited version of an article accepted for publication/published in Modelling and Simulation in Materials Science and Engineering. IOP Publishing Ltd is not responsible for any errors or omissions in this version of the manuscript or any version derived from it. The Version of Record is available online at http://dx.doi.org/10.1088/0965-0393/23/8/085008. Citation details: Leyson, G. P. M.; Curtin, W. A. Model. Simul. Mater. Sci. Eng. 2016, 24 (6), 65005.

Robust Atomistic Calculation of Dislocation Line Tension

\begin{tabular}{lcr}
\hline Elasticity & Isotropic $(\mathrm{eV} / \AA)$ & Anisotropic $(\mathrm{eV} / \AA)$ \\
\hline Edge $^{\text {Ercolessi-Adams }}$ & 0.060 & 0.065 \\
Edge $^{\text {Mishin }}$ & 0.052 & 0.055 \\
Screw $^{\text {Ercolessi-Adams }}$ & 0.258 & 0.266 \\
Screw $^{\text {Mishin }}$ & 0.238 & 0.244 \\
\hline MD (guide to the eye) & & \\
\hline Edge $^{\text {atom, Ercolessi-Adams }}$ & & 0.072 \\
Screw $^{\text {atom, Ercolessi-Adams }}$ & & 0.139 \\
\hline
\end{tabular}

Table 2. $\ln (L)$ prefactor, $\mathrm{C}_{1}$, of the dislocation line tension $\Gamma$ for the ErcolessiAdams [59] and Mishin [61] interatomic potentials, using both isotropic and anisotropic elasticity. Also shown is the value obtained and plotted (atom, dashed in figure 5) from the present atomistic study for each case.

only on the character of the nominally straight dislocation but also on its orientation with respect to the lattice $[54,55]$. For Al, we have computed Eq. 4 for both isotropy and general anisotropy (detailed in the appendix) using the full elastic stiffness tensors corresponding to the Ercolessi-Adams [59] and Mishin [61] interatomic potentials at 0K, respectively. Table 2 shows the $\ln (L)$ prefactor $\mathrm{C}_{1}$. Al is relatively isotropic so that the effects of anisotropy are relatively small $(\leq 10 \%)$.

Finally, fcc dislocations dissociate into partial dislocations, so it is possible that the line tension for the full dislocation might also be considered as due to two interacting partial dislocations. Since the line tension of an individual dislocation scales as $b^{2}$, it is unclear whether the line tension of two interacting partial dislocations with burgers vectors $b_{p}=b \sqrt{3} / 3$ is equal to that of a full dislocation of burgers vector $b$. This problem is analytically tractable within isotropic elasticity, and the result shows that the line tension for two partial dislocations depends on the partial spacing $d$ divided by the obstacle spacing $L$. For partial dislocations in $\mathrm{Al}, d \sim 1 \mathrm{~nm}$ and $d / L<<1$ for relevant dislocation lengths of $L>30 \mathrm{~nm}$, so that the line tension is essentially equal to that of the full undissociated $(d=0)$ dislocation.

\section{Molecular Dynamics Simulation and Analysis to Compute Line Tension}

\subsection{Simulation Details}

Low temperature (1K) Molecular Dynamics (MD) simulations were performed using a multiscale method that couples a small atomistic region $(\sim 1$ million atoms $)$ to a large surrounding continuum region. The method uses the standard large-scale parallel atomistic simulation software LAMMPS [41] in conjunction with a parallelized finite element scheme developed for large 3D multiscale simulations [52]. The coupling between atomistic and continuum regions creates zero errors when dislocations are more than $\approx 1 \mathrm{~nm}$ from the atom/continuum interface, and the very large surrounding continuum domain eliminates all spurious image forces that would otherwise arise due 
This is an author-created, un-copyedited version of an article accepted for publication/published in Modelling and Simulation in Materials Science and Engineering. IOP Publishing Ltd is not responsible for any errors or omissions in this version of the manuscript or any version derived from it. The Version of Record is available online at http://dx.doi.org/10.1088/0965-0393/23/8/085008. Citation details: Leyson, G. P. M.; Curtin, W. A. Model. Simul. Mater. Sci. Eng. 2016, 24 (6), 65005.

to interactions of the curved dislocations with the outer boundaries of the sample [50]. Thus, no corrections for boundary conditions are needed.

The simulation cell is a single crystal fcc sample. Different fcc lattice orientations are used for studies involving edge or screw dislocations, but always maintaining the dislocation line along the periodic $\mathrm{Y}$ direction. For all cases, $\mathrm{Z}=\left[\begin{array}{lll}1 & 1 & 1\end{array}\right]$ is normal to the dislocation glide plane, while $\mathrm{X}$ and $\mathrm{Y}$ (glide and line) directions are $\left[\begin{array}{lll}1 & \overline{1} & 0\end{array}\right]\left[\begin{array}{lll}1 & 1 & \overline{2}\end{array}\right]$ and $\left[\begin{array}{lll}1 & 1 & \overline{2}\end{array}\right]\left[\begin{array}{lll}1 & \overline{1} & 0\end{array}\right]$ for edge and screw dislocations, respectively. The atomistic portion of the much-larger simulation cell has dimensions of $\mathrm{X} \approx 18 \mathrm{~nm}, \mathrm{Y} \approx 13-52 \mathrm{~nm}$ (varying dislocation line length), and $\mathrm{Z} \approx 10 \mathrm{~nm}$. The $\mathrm{Al}$ interatomic potentials developed by Ercolessi and Adams [59] and by Mishin et al [61] describe the nonlinear interactions between atoms. We insert a single initially-straight dislocation into the atomistic region by displacing atoms and nodes of the multiscale model according to the singular Volterra solution and external applied boundary conditions as specified in [52]. The inner nodes and atoms are allowed to relax using explicit dynamics at $1 \mathrm{~K}$, during which the singular dislocation core dissociates into two partials. A damping stadium method is used to control the temperature in the atomistic region. The obstacles are then created by identifying small cubic volumes of $\sim 1 \mathrm{~nm}^{3}$ encompassing the dislocation core that are then held fixed during subsequent simulations. Spurious stress fields created by these rigid obstacles scale with the applied load and can be approximated to leading order as $\tau_{o b s}<\frac{\tau_{a p p}}{32}\left(\frac{a}{R}\right)^{3}$, where $a=1 \mathrm{~nm}$ is the characteristic obstacle length and $R$ is the distance from the obstacle. For all simulation results reported here, $\tau_{\text {obs }}(\mathrm{R}>1.5 \mathrm{~nm})<3$ $\mathrm{MPa}$ and we consider its influence on bowing out of the dislocation over scales of $\geq 10 \mathrm{~nm}$ to be negligible. A homogeneous resolved shear stress $\tau_{a p p}\left(\tau_{x z}\right.$ for the edge, $\tau_{y z}$ for the screw) is applied using a homogeneous shear strain $\epsilon$ and the appropriate shear modulus $\mu=\left(C_{44}+C_{11}-C_{12}\right) / 3$ so that $\tau_{a p p}=\mu \epsilon$. Upon loading, the (pinned) dislocation bows out. Inertial effects [42] are avoided by using small loading increments of $\Delta \tau_{a p p} \approx 5 \mathrm{MPa}$ to obtain essentially quasi-static equillibrium shapes. The dislocation structure (partial dislocations, stacking fault) were detected using common neighbor analysis (CNA) and AtomEye [62] for visualization.

\subsection{Configurational Analysis}

The outcome of the MD simulation for a given $L$ and $\tau_{a p p}$ is a bowed-out dislocation configuration. Evaluation of the configurational derivative (i.e. $\mathrm{d} A / \mathrm{d} S$ ) appearing in eq. 3 first requires measurement of the total length of dislocation line, $S$, and the area enclosed by the bow-out, $A$. Using a moving average procedure, each partial dislocation is detected using CNA and its line is divided into discrete segments (shown in red in figure 1). The two partial dislocations are then averaged to create a single, continuous curve (shown in green in figure 1). The line length $S$ is the sum of the lengths of the discrete segments and the area $A$ is calculated using Simpsons rule. There is no assumption about the overall shape of the dislocation. We have verified that the stacking fault area is unchanged during the bowing-out process, although there are 
This is an author-created, un-copyedited version of an article accepted for publication/published in Modelling and Simulation in Materials Science and Engineering. IOP Publishing Ltd is not responsible for any errors or omissions in this version of the manuscript or any version derived from it. The Version of Record is available online at http://dx.doi.org/10.1088/0965-0393/23/8/085008. Citation details: Leyson, G. P. M.; Curtin, W. A. Model. Simul. Mater. Sci. Eng. 2016, 24 (6), 65005.

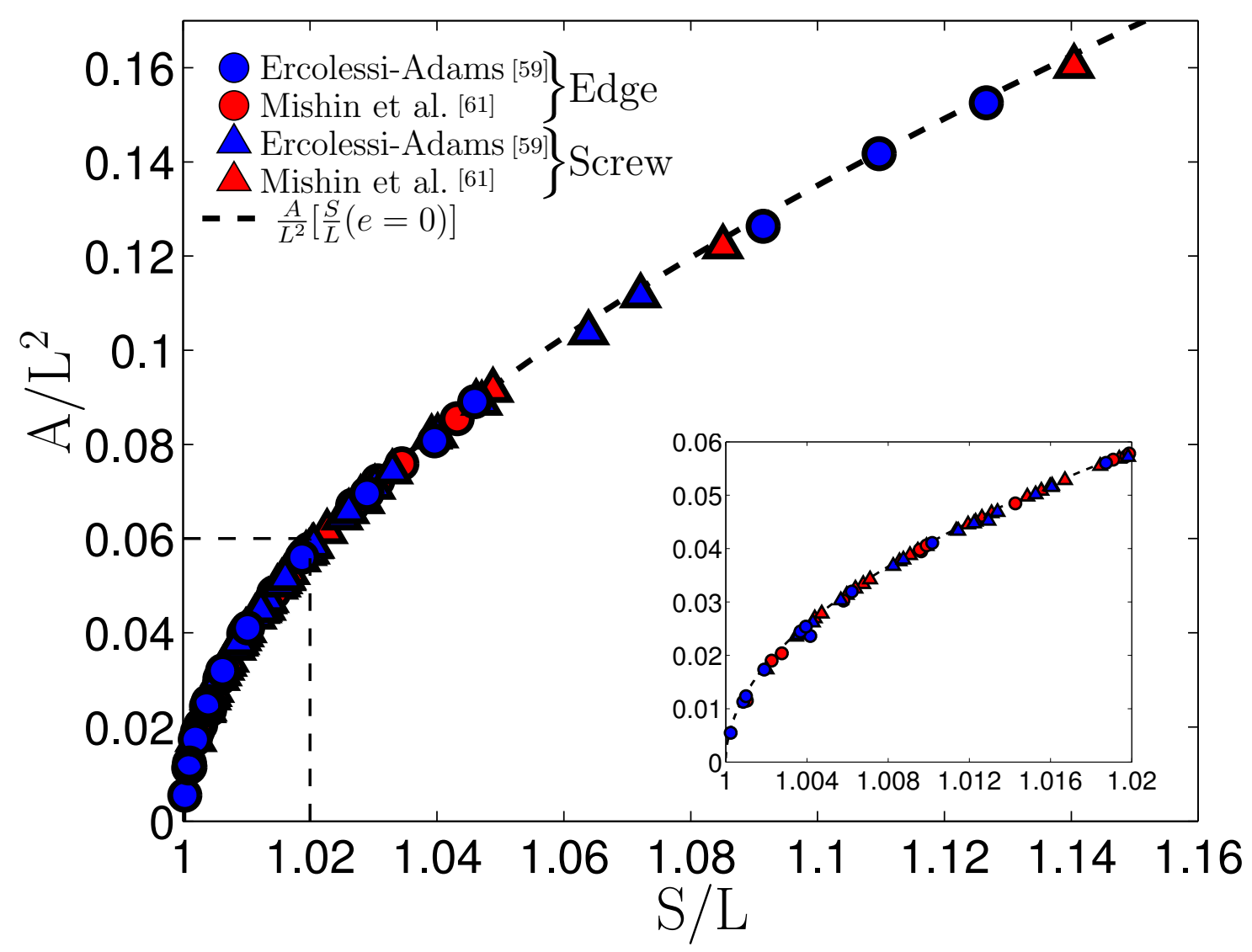

Figure 2. Normalized area enclosed by the bow-out $\left(\frac{A}{L^{2}}\right)$ versus normalized line length $\left(\frac{S}{L}\right)$ for each bow-out configuration. Also shown (--) is the relationship between $A$ and $S$ used in calculating $R$. Inset: Normalized area enclosed by the bow-out versus normalized line length for $\frac{A}{L^{2}}<0.06$ and $\frac{S}{L} \leq 1.02$

small differences in the partial separation of $\pm b_{p}$ along the dislocation line associated with slightly different lateral positions of CNA-visualized "kinks" along the two partials.

Evaluation of the derivative $d A / d S$ is difficult to perform accurately by purely numerical means based on a sequence of bow-out configurations. We thus develop a general analytic model to assist with this differentiation, as follows. We assume that the dislocation bows-out into a curved geometry with a non-constant radius (as discussed and detailed in [63]), which is expected due to the difference in line tension for screw and edge dislocations. There is no analytic closed-form relationship between the length $\mathrm{S}$ and area $\mathrm{A}$ associated with a general arc having a chord length L. However, for the small bow-out problem of interest, where $L$ is much less than the minimum radius (i.e. $S / L \approx 1$ ), the relationship between $A$ and $S$ is almost independent of the non-constant radius and thus essentially equal to that of a circular arc. In the limit of an ellipse (with eccentricity, e), it can be readily verified that for an elliptical arc and a circular arc of 
This is an author-created, un-copyedited version of an article accepted for publication/published in Modelling and Simulation in Materials Science and Engineering. IOP Publishing Ltd is not responsible for any errors or omissions in this version of the manuscript or any version derived from it. The Version of Record is available online at http://dx.doi.org/10.1088/0965-0393/23/8/085008. Citation details: Leyson, G. P. M.; Curtin, W. A. Model. Simul. Mater. Sci. Eng. 2016, 24 (6), 65005.

equal arc length $S$ and bisected length $L$, the difference in area A is negligibly small,

$$
(A[S(e)]-A[S(e=0)]) / L^{2} \approx \mathcal{O}\left(\frac{e L}{R_{m a j}}\right)^{2}
$$

Using the circle radius $\mathrm{R}$ as a parametric variable, the area $A$ versus arc length $S$ can be solved by eliminating $R$ from the two equations

$$
S=2 R \sin ^{-1}\left(\frac{L}{2 R}\right), \quad A=\left(R^{2} \sin ^{-1}\left(\frac{L}{2 R}\right)-\frac{R L}{2} \sqrt{1-\frac{L^{2}}{4 R^{2}}}\right)
$$

Figure 2 shows the resulting relationship between $A / L^{2}$ and $S / L$, the accuracy of which we have also validated with explicit numerical computations on ellipses. Also shown in figure 2 are our simulation results for both edge and screw dislocations for a range of $L$ and a range of $\tau_{a p p}$. All of our data fall on the analytic line of $A / L^{2}$ versus $S / L$, indicating that the measured shapes (whatever they may be) are consistent with elliptical arcs in terms of the relationship between $S / L$ and $A / L^{2}$, despite not having actually quantified the shapes. For any individual data point lying on this "universal" curve of $A / L^{2}$ versus $S / L$, we can accurately calculate the derivative $d A / d S$ using the universal curve at the given value of $A$ and $S$. There is then no need for finite-difference derivatives between numerically-simulated configurations that would be inaccurate due to small numerical noise in our measurements of $A$ and $S$.

\subsection{Effective Peierls stress}

Due to the discrete atomistic lattice, dislocations encounter a periodic resistance to motion with a wavelength of $b$ that is the Peierls stress $\tau_{P}$. The influence of $\tau_{P}$ must be considered when evaluating the line tension. The line tension is primarily a continuum concept applied to a smooth continuous dislocation line. Within a similar continuum framework, the discrete features giving rise to $\tau_{P}$ at the scale of $b$ are neglected in favor of a continuous resistance of $\tau_{P}$ acting at all points along a dislocation line to oppose motion. Thus, under a true applied stress $\tau_{a p p}$, the effective applied stress, in the sense of a Peach-Koehler [64] force, $\tau_{\text {app,eff }}$ acting on a straight dislocation is taken as

$$
\tau_{\text {app }, e f f}=\tau_{\text {app }}-\operatorname{sgn}\left(\Delta \tau_{\text {app }}\right) \tau_{P}
$$

where $\Delta \tau_{a p p}$ denotes the difference in applied load between the current and the previous loading increment and the $s g n$ term ensures that the Peierls stress opposes the dislocation motion in the direction of $\Delta \tau_{a p p}$. The Peierls stress thus leads to a hysteresis in the response of a dislocation as it glides forward and backward. The implication for dislocation bow-out is that during the bowing out process occuring during loading, the effective applied stress should correspond to $\tau_{a p p, e f f}=\tau_{a p p}-\tau_{P}$. If, after some amount of bow-out, the applied load is then reduced, the dislocation should retreat but the configurations should correspond to an effective applied stress $\tau_{a p p, e f f}=\tau_{a p p}+\tau_{P}$ since the Peierls stress will oppose the retreating motion. Therefore, at any position, the difference in applied stresses required to move the dislocation forward and to move 
This is an author-created, un-copyedited version of an article accepted for publication/published in Modelling and Simulation in Materials Science and Engineering. IOP Publishing Ltd is not responsible for any errors or omissions in this version of the manuscript or any version derived from it. The Version of Record is available online at http://dx.doi.org/10.1088/0965-0393/23/8/085008. Citation details: Leyson, G. P. M.; Curtin, W. A. Model. Simul. Mater. Sci. Eng. 2016, 24 (6), 65005.

Robust Atomistic Calculation of Dislocation Line Tension

\begin{tabular}{lccr}
\hline & $\begin{array}{c}\tau_{\text {Peierls }, 0 K} \\
(\mathrm{MPa})\end{array}$ & $\begin{array}{c}\tau_{\text {Peierls }, 1 K} \\
(\mathrm{MPa})\end{array}$ & $\begin{array}{r}\tau_{P, \text { eff }} \\
(\mathrm{MPa})\end{array}$ \\
\hline Edge $^{\text {Ercolessi-Adams }}$ & 2.4 & $<2$ & 0.25 \\
Edge $^{\text {Mishin }}$ & 2 & $<2$ & 0.1 \\
Screw $^{\text {Ercolessi-Adams }}$ & 14 & 9 & 0.6 \\
Screw $^{\text {Mishin }}$ & 33 & 30 & 3.6 \\
\hline
\end{tabular}

Table 3. Measured Peierls stress of straight and curved dislocations. The $\tau_{P, e f f}$ values are taken directly from the converged values of the atomistic simulations with sufficiently high kink density (see text and figure 4), and are used in the subsequent calculation of $\Gamma$ in figure 5 .

the dislocation backward is $2 \tau_{P}$. In computing the line tension, the appropriate $\tau_{a p p, e f f}$ must be used along with the dislocation configuration (and associated $d A / d S$ ).

The Peierls stress $\tau_{P}$ is calculated atomistically for straight dislocations as the applied stress needed to initiate dislocation flow in an infinite material, and depends on the dislocation character $\phi$. The Peierls stresses for the edge and screw dislocations for the Ercolessi-Adams and Mishin potentials are shown in table 3, as measured at both $T=0 K$ and $T=1 K$ with the differences being small. As is well-known, $\tau_{P}$ for the $\mathrm{Al}$ edge is quite small, $\sim 2 \mathrm{MPa}$, while $\tau_{P}$ for the screw is non-negligible being on the scale of applied stresses needed to bow-out dislocations and varies between the two potentials. Focusing on the screw dislocation, if these values of $\tau_{P}$ for the perfectly straight dislocation are applied to the bow-out problem, then at any given configuration the applied stress to move a bow-out screw forward should be larger than the stress to retract the bowed-out screw by either $18 \mathrm{MPa}$ or $60 \mathrm{MPa}$, for the EA and Mishin cases at $T=1 K$ respectively. Simulations of the motion of bowed-out screw dislocations show no such large hysteresis. Figure 3 shows loading and unloading configurations corresponding to the same applied load $\tau_{a p p}$, for several values, achieved during loading up to $168 \mathrm{MPa}$ and then unloading, in small load increments. The differences in configurations of the dislocation during loading and unloading at the same applied stress $\tau_{a p p}$ are nearly the same, differing by only $\sim 2 b$ at the peak of the bowout. Although the initially straight dislocation requires an applied load greater than $\tau_{P}$ to begin bowing, the dislocation bowing for moderately-curved dislocations does not correspond or conform to the behavior predicted using eq. 9 with $\tau_{P}$ for the straight dislocation. In other words, the measured $\tau_{P}$ for a straight dislocation cannot be used as the Peierls stress for a curved dislocation.

The loading and unloading configurations are slightly different, with the unloading configuration always ahead of the loading configuration (by $\sim 2 b$ ) at the same applied stress. This indicates that there is some effective Peierls stress $\tau_{P, \text { eff }}$ acting on the curved dislocations that is much lower than the Peierls stress acting on straight dislocations $\left(\tau_{P}\right)$. To deduce the value of $\tau_{P, \text { eff }}$ for such a continuum curved dislocation, we assume that $\tau_{P, e f f}$ acts as a continuum Peierls stress, similar to $\tau_{P}$, to oppose the 
This is an author-created, un-copyedited version of an article accepted for publication/published in Modelling and Simulation in Materials Science and Engineering. IOP Publishing Ltd is not responsible for any errors or omissions in this version of the manuscript or any version derived from it. The Version of Record is available online at http://dx.doi.org/10.1088/0965-0393/23/8/085008. Citation details: Leyson, G. P. M.; Curtin, W. A. Model. Simul. Mater. Sci. Eng. 2016, 24 (6), 65005.

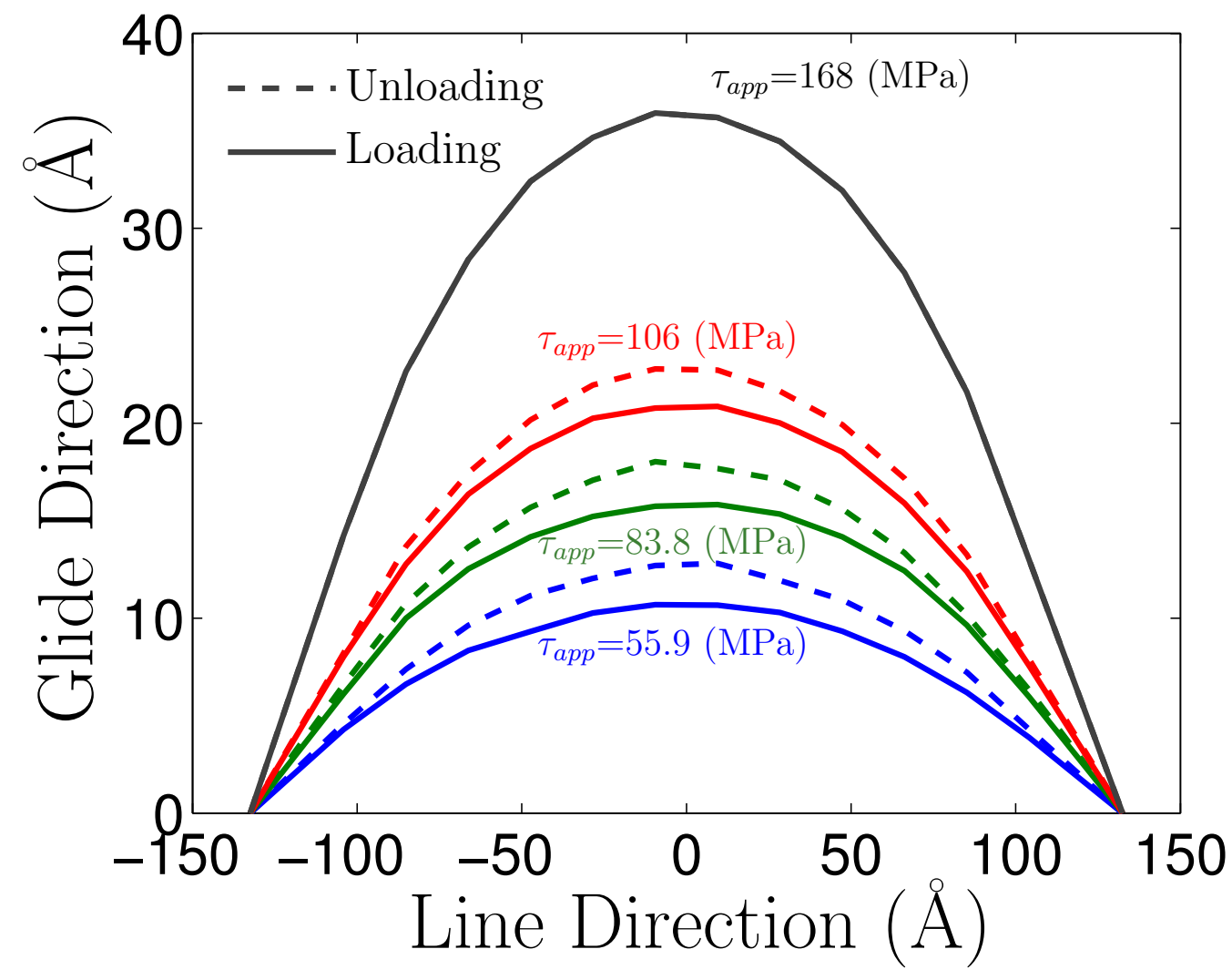

Figure 3. Hysteresis in the bow-out of the Mishin [61] initially screw dislocation at three applied loads. The amount of bow-out for loading (solid, $\Delta \tau_{a p p}>0$ ) is only slightly less than the amount of bow-out for unloading (dashed, $\Delta \tau_{a p p}<0$ ). Also shown is the configuration at the peak load of $\tau_{a p p}=168 \mathrm{MPa}$ from which point the unloading commenced.

dislocation motion according to eq. 9. For any pair of loading and unloading bow-out configurations $\left(A_{l}, S_{l}, R_{l}\right)$ and $\left(A_{u}, S_{u}, R_{u}\right)$ with the associated parametric variable, $R$ (from eqs. 8) measured at the same applied stress, we assume that the line tension $\Gamma$ is the same for the two configurations. The two configurations must then be related through eq. 3 and eq. 9, leading to a system of two linear equations that when solved for $\tau_{P, e f f}$ is

$$
\tau_{P, e f f}=\tau_{a p p}\left(\frac{1-\frac{R_{u}}{R_{l}}}{1+\frac{R_{u}}{R_{l}}}\right)
$$

Inserting the effective Peierls stress (eq. 10) applicable to curved dislocations in to eq. 9 leads to an effective applied stress for the bow-out dislocations considered in this study:

$$
\tau_{a p p, e f f}=\tau_{a p p}\left(1-\operatorname{sgn}\left(\Delta \tau_{a p p}\right)\left(\frac{1-\frac{R_{u}}{R_{l}}}{1+\frac{R_{u}}{R_{l}}}\right)\right)
$$

This definition of $\tau_{P, e f f}$ enforces equality of both $\Gamma$, and $\tau_{\text {app,eff }}$ for two configurations at the same applied load with the same spacing between obstacles $(L)$, independent of 
This is an author-created, un-copyedited version of an article accepted for publication/published in Modelling and Simulation in Materials Science and Engineering. IOP Publishing Ltd is not responsible for any errors or omissions in this version of the manuscript or any version derived from it. The Version of Record is available online at http://dx.doi.org/10.1088/0965-0393/23/8/085008. Citation details: Leyson, G. P. M.; Curtin, W. A. Model. Simul. Mater. Sci. Eng. 2016, 24 (6), 65005.

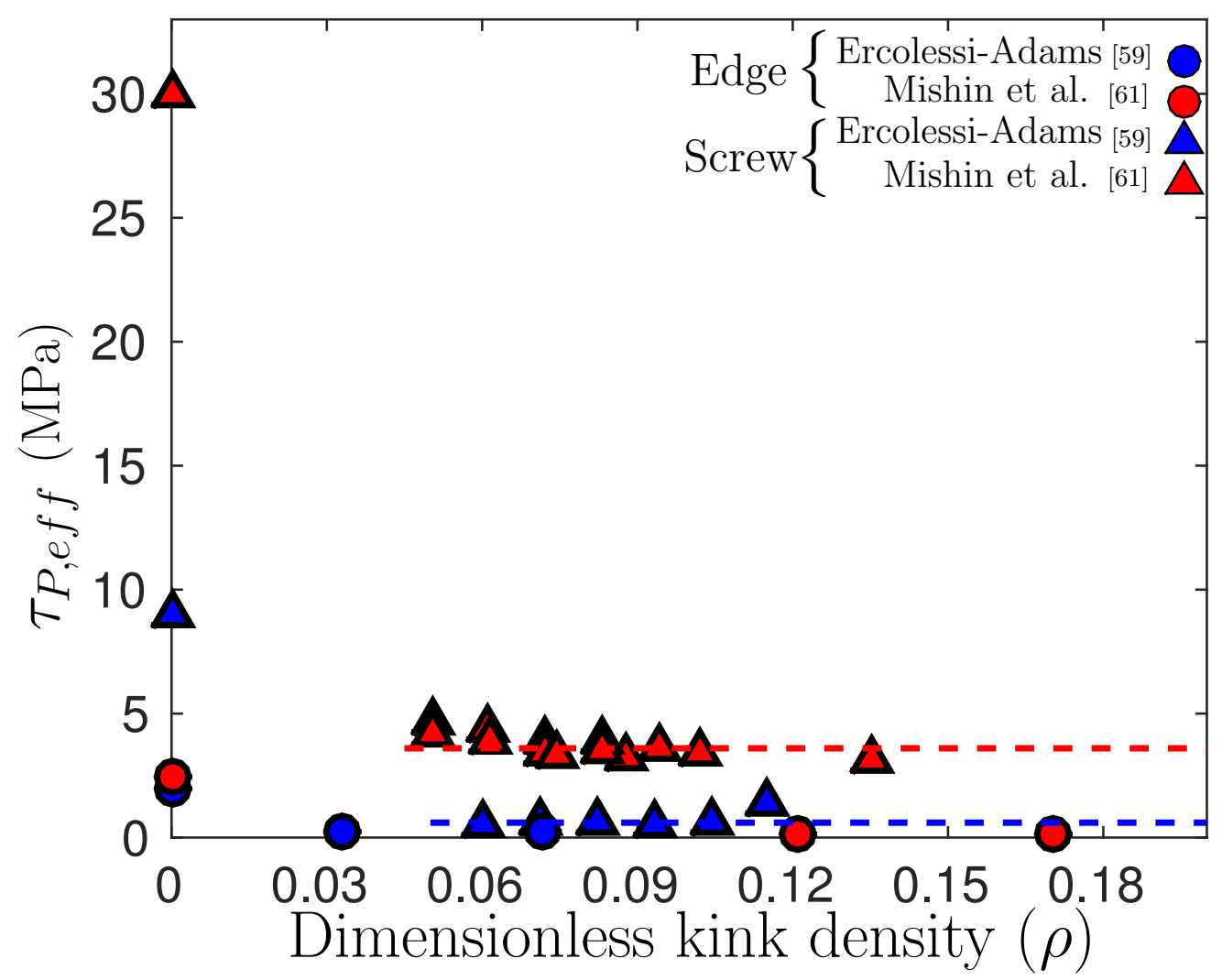

Figure 4. Effective Peierls stress, $\tau_{P, e f f}$, versus dimensionless kink density $(\rho \propto h / L)$ in the bowed-out configuration, for both nominally edge and screw dislocations. The dimensionless kink density is related to the peak bow-out distance $h$ as $\rho=2 h / L$ and $2 \sqrt{3} h / 3 L$ for edge and screw, respectively. The converged $\tau_{P, \text { eff }}$ values for both the Ercolessi-Adams and Mishin nominally screw dislocations are shown as blue and red dashed lines respectively at $\tau_{P, \text { eff }}=0.6$ and $3.6 \mathrm{MPa}$, and are tabulated in table 3 along with those for straight dislocations.

$\operatorname{sgn}\left(\Delta \tau_{a p p}\right)$. The computed $\tau_{P, e f f}$ for a number of loading/unloading configurations (various $L$ and $\tau_{a p p}$ ), for both edge and screw dislocations, is shown in figure 4 . In figure $4, \tau_{P, \text { eff }}$ is shown versus the dimensionless kink density, $\rho^{\text {edge }}=2 h / L$ or $\rho^{\text {screw }}=2 \sqrt{3} h / 3 L$, required to achieve the peak bow-out $h$ in each configuration. Each data point is completely independent. For dislocations with a low kink density (small bow-out relative to the dislocation length), $\rho<0.05$, the discrete nature of the dislocation bow-out makes a smooth continuum description inappropriate. This is manifested in, among other ways, an inability to measure an effective Peierls stress for very low kink densities $\rho \leq 0.05$, and so no data is shown. However, for larger bowout or modestly-curved dislocations, $\rho \geq 0.05$, an effective Peierls stress $\tau_{P, e f f}<<\tau_{P}$ emerges that is nearly independent of configuration (independent of $\rho, \mathrm{L}, \tau_{a p p}$ ) and thus represents an appropriate "continuum-level" Peierls stress for the curved dislocation. 
This is an author-created, un-copyedited version of an article accepted for publication/published in Modelling and Simulation in Materials Science and Engineering. IOP Publishing Ltd is not responsible for any errors or omissions in this version of the manuscript or any version derived from it. The Version of Record is available online at http://dx.doi.org/10.1088/0965-0393/23/8/085008. Citation details: Leyson, G. P. M.; Curtin, W. A. Model. Simul. Mater. Sci. Eng. 2016, 24 (6), 65005.

For the edge dislocations, which have small $\tau_{P}$, the effective $\tau_{P, \text { eff }}$ is nearly zero for both interatomic potentials. For the screw dislocations, which have larger $\tau_{P}$, the effective $\tau_{P, \text { eff }}$ is small, typically $\sim 0.5 \mathrm{MPa}$ for the EA potential and $\sim 3.6 \mathrm{MPa}$ for the Mishin potential. The consistency of the deduced value of $\tau_{P, \text { eff }}$ across a range of configurations, for each initial character and interatomic potential, supports the notion that a single value of continuum (effective) Peierls stress can be considered to operate for moderately curved dislocations. These effective values are appropriate at the temperature of our simulations, $T=1 K$ and are tabulated in the final column of table 3 .

\section{Results}

In the previous section, we presented the essential pieces of the simulation and analysis needed to compute $\Gamma$. For an initial length $L$, we have measured $A / L^{2}$ and $S / L$ at various values of $\tau_{a p p}$ (figure 2). We have also deduced the effective applied stress $\tau_{\text {app }, \text { eff }}=\tau_{\text {app }} \pm \tau_{P, \text { eff }}$ acting on the bowed-out dislocation in the presence of the effective Peierls stress (figure 4). For each configuration, our analytic analysis for the geometry of $A / L^{2}$ versus $S / L$ yields the value of $d A / d S$ necessary to obtain $\Gamma$. In light of the predictions of elasticity theory (eq. 4), we present the computed line tension $\Gamma$ versus $\ln (L / b)$, as shown in figure 5 for the two interatomic potentials [59,61], three obstacle spacings $L$, and initially edge and screw dislocations. For moderate bow-outs $\rho \geq 0.05$ where the continuum description is valid, the results are independent of the amount of bow-out to leading order in $h / L$ (e.g. $S, A$, and/or $h$ ) and therefore the derived line tension is a function primarily of obstacle spacing $(\mathrm{L})$ and dislocation character $(\phi)$. The atomistic computation of the line tension $(\Gamma)$ is the first main result of this paper.

The line tension $\Gamma_{\text {edge }}$ for the edge dislocation is quite consistent between the two potentials, with the Mishin potential yielding slightly smaller values, $\approx-0.05 \mathrm{eV} / \AA$. Both potentials show a good linear scaling in $\ln (L / b)$ with similar slopes. The line tension for the screw dislocation is larger than that for the edge, as expected, and both potentials predict comparable values for $\Gamma_{\text {screw }}$, with the Mishin potential greater by $\approx 0.05 \mathrm{eV} / \AA$ at the smallest and largest sizes, with twice that difference at the intermediate length $L / b \approx 90$. The results for the EA potential again show a good linear scaling in $\ln (L / b)$ while the Mishin potential results are inconclusive about a $\ln (L / b)$ scaling. The Mishin screw has a rather high Peierls stress for the straight dislocation, suggesting that the potential may have some inaccurate features that are not easily identifiable but are reflected in the Peierls stress and also influence the bowing and the line tension. Here, we thus focus attention on the consistent EA results, while retaining the knowledge that the general magnitudes of $\Gamma$ when comparing the EA and Mishin potentials are quite comparable for both edge and screw, over this range of $L$. Our results for the atomistic line tension for the EA potential can be fit to the form of elasticity plus a core energy term,

$$
\Gamma^{a t o m}=C_{1}^{a t o m} \ln (L / b)+C_{0}^{a t o m}
$$


This is an author-created, un-copyedited version of an article accepted for publication/published in Modelling and Simulation in Materials Science and Engineering. IOP Publishing Ltd is not responsible for any errors or omissions in this version of the manuscript or any version derived from it. The Version of Record is available online at http://dx.doi.org/10.1088/0965-0393/23/8/085008. Citation details: Leyson, G. P. M.; Curtin, W. A. Model. Simul. Mater. Sci. Eng. 2016, 24 (6), 65005.

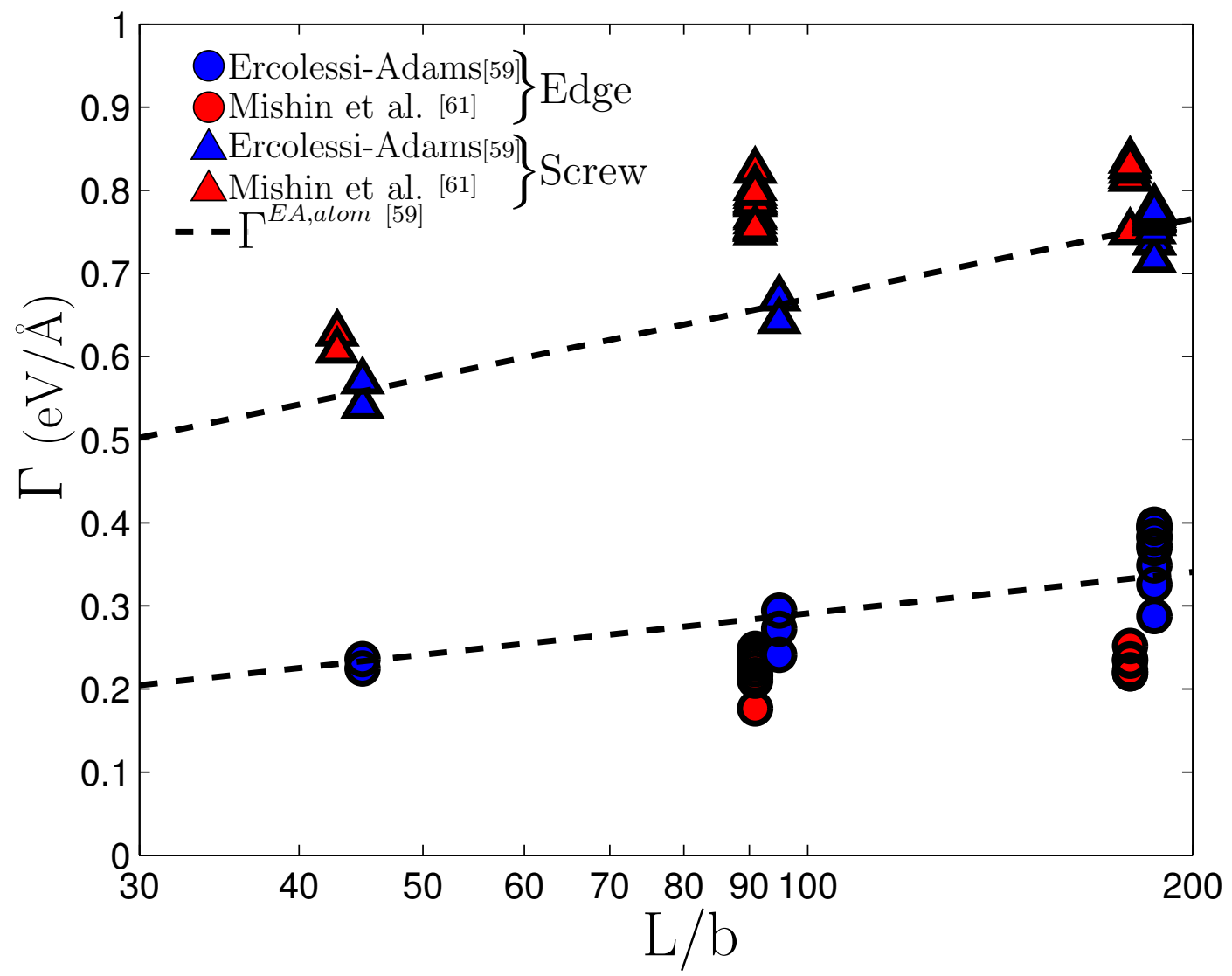

Figure 5. $\Gamma$ versus $\ln (L / b)$ for the interatomic potentials of Ercolessi-Adams [59] (blue) and Mishin [61] (red) for nominally edge (circles) and screw (triangles) dislocations. The data collapses well, indicating the robustness of the method. Both data sets have been shifted by $\sim 2$ Burgers vectors from their true $L / b$ values to improve clarity of the figure. Dashed lines show fits to the form $\Gamma=C_{1}^{\text {atom }} \ln (L / b)+E_{\text {core }}^{\text {atom }}$ for the Ercolessi-Adams potential, yielding $\Gamma_{\text {edge }}^{E A, a t o m}=0.072 \ln (L / b)-0.04(\mathrm{eV} / \AA)$ and $\Gamma_{\text {screw }}^{E \text { A,atom }}=0.139 \ln (L / b)+0.03(\mathrm{eV} / \AA)$.

The variations in $\Gamma$ for one potential at one length $L$ reflect the level of accuracy of the measurements of $A, S$, and $\tau_{P, e f f}$. Numerical measurements of $A$ versus $S$ for various $L$ for perfect ellipses with moderate ellipticity $e<0.7$ show near-perfect agreement with the analytic result for the circle $A[S(e=0)]$ curve. Thus, deviations in our results are due to the fact that the dislocations are not smooth lines, which leads to associated small numerical errors in general. For very small bow-outs, both the evolution of the bow-out shape (i.e. $d A / d S$ ) and the effective Peierls stress are ill-defined and so our analyses and results are restricted to the domain $\rho>0.05$. In this regime, a continuous smooth bow-out description is applicable, a configuration-independent effective Peierls stress can be obtained (see figure 4 ), and a reliable $\Gamma$ computed. For the edge dislocation, we obtain $C_{1}^{\text {atom }}=0.072 \mathrm{eV} / \AA$ and $C_{0}^{\text {atom }}=-0.04 \mathrm{eV} / \AA$. For the screw dislocation, we obtain $C_{1}^{\text {atom }}=0.139 \mathrm{eV} / \AA$ and $C_{0}^{\text {atom }}=+0.03 \mathrm{eV} / \AA$. 
This is an author-created, un-copyedited version of an article accepted for publication/published in Modelling and Simulation in Materials Science and Engineering. IOP Publishing Ltd is not responsible for any errors or omissions in this version of the manuscript or any version derived from it. The Version of Record is available online at http://dx.doi.org/10.1088/0965-0393/23/8/085008. Citation details: Leyson, G. P. M.; Curtin, W. A. Model. Simul. Mater. Sci. Eng. 2016, 24 (6), 65005.
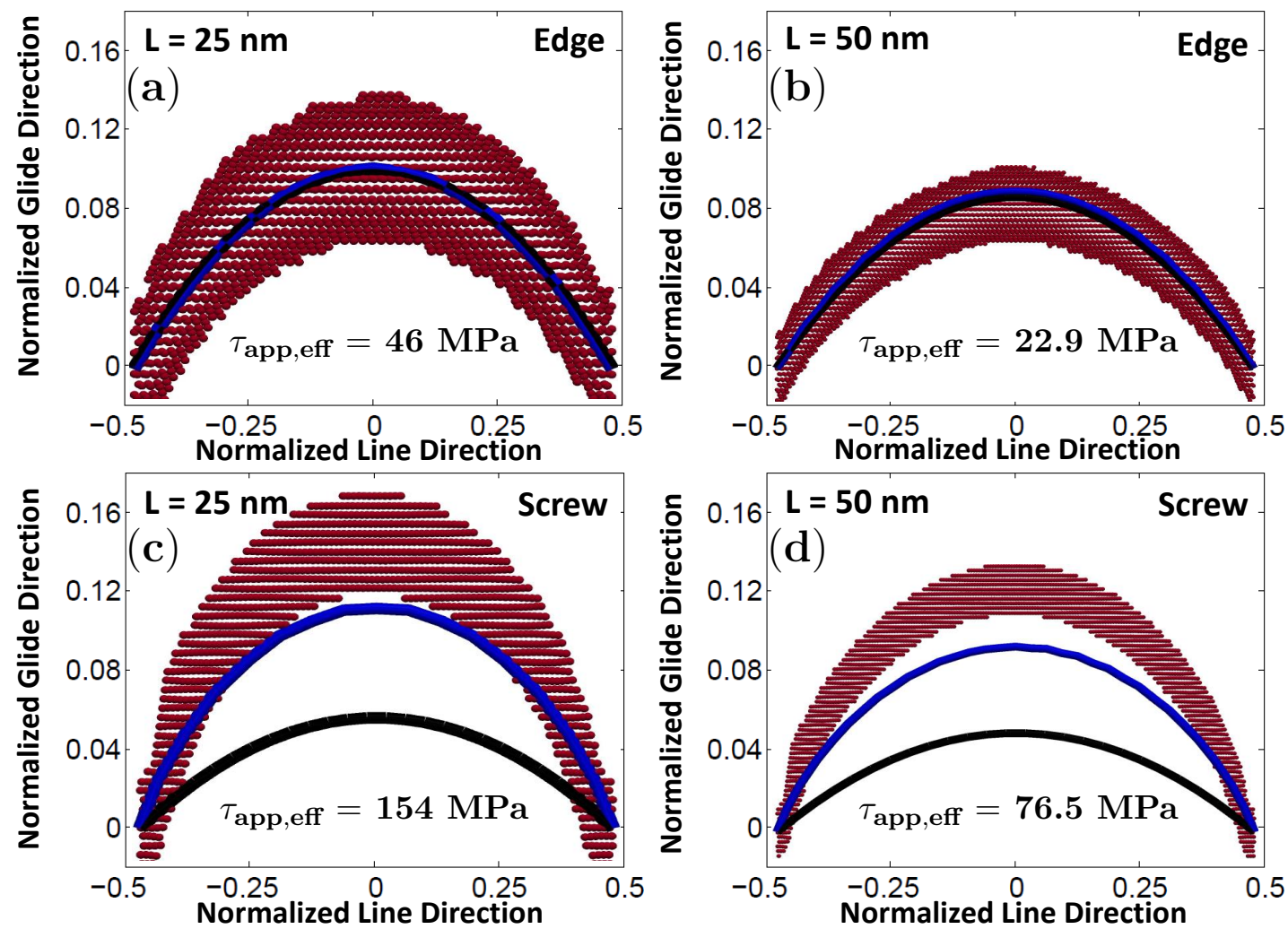

Figure 6. Configurations of four bowed-out dislocations of lengths $\mathrm{L}=25 \mathrm{~nm}$ (left) and $\mathrm{L}=50 \mathrm{~nm}$ (right) for both the nominally edge (top) and screw dislocation (bottom), as computed via MD (red), via Non-Singular (NS) Theory [37] (blue) and via classical singular elasticity [22] (black). The MD computations were performed with the Ercolessi-Adams [59] interatomic potential; The NS Theory computations were performed using ParaDiS [37] with both $r_{c} / b=2.718$ and $E_{\text {core }}=\frac{2.03 \mu}{4 \pi}$ fixed for each simulation; The classical singular theory computations were performed with a cut-off radius of $r_{0} / b=1$ and $E_{\text {core }}=\frac{4.83 \mu}{4 \pi}$ fixed throughout the comparison. All cut-off parameters were selected to most accurately reproduce the $25 \mathrm{~nm}$ edge dislocation (top left). In all four comparisons the effective applied stress ( $\left.\tau_{a p p, e f f}\right)$ extracted from the MD simulations was used in the DDD simulations and is shown within each figure.

\section{Discussion}

We start with discussion of the $C_{1}$ coefficient, which has an unambiguous value within elasticity theory. For the edge, $C_{1}^{\text {atom }} \approx C_{1}^{\text {aniso }}$, within the uncertainty of our measurements. For the screw, however, $C_{1}^{\text {atom }}<<C_{1}^{\text {aniso }}$, differing by a factor of $C_{1}^{\text {aniso }} / C_{1}^{\text {atom }} \approx 2$, which is not negligible. The $C_{1}$ term arises in elasticity theory due to the change in self-energy (due to both rotation, and elongation) of each infinitesimal segment as the dislocation bows-out. It therefore would seem sensible that elasticity should apply. Our failure to match anisotropic elasticity might cast doubt on the validity of the atomistic simulation and results but the results are very robust and our final values for $\Gamma$ are very consistent across different amounts of bow-out and applied stress. 
This is an author-created, un-copyedited version of an article accepted for publication/published in Modelling and Simulation in Materials Science and Engineering. IOP Publishing Ltd is not responsible for any errors or omissions in this version of the manuscript or any version derived from it. The Version of Record is available online at http://dx.doi.org/10.1088/0965-0393/23/8/085008. Citation details: Leyson, G. P. M.; Curtin, W. A. Model. Simul. Mater. Sci. Eng. 2016, 24 (6), 65005.

Furthermore, the bowed-out atomistic dislocation is not a smooth continuous line in the sense assumed within the elastic theory or within standard discrete dislocation modeling [22]. Instead the atomistic dislocation consists of a displacement discontinuity profile along the slip-plane which evolves as the dislocation bows-out in response to the applied stress and to the underlying landscape of the generalized stacking fault energy surface. The long-ranged elastic fields arise from the gradient of the displacement discontinuity profile along the slip plane within the dislocation core. Here, we are studying evolving curved dislocations, complete with Shockley partial dislocations separated by a stacking fault, that might also be characterized in terms of dislocation segments and kinks, and so it is perhaps surprising that a $\ln (L / b)$ scaling emerges at all. In fact, we have performed DD computations of $\Gamma$ by representing the bowed-out dislocation by a discrete set of dislocation segments with each segment having a character chosen from only a few high symmetry orientations $(0,30,60,90)$ similar in spirit to those examined by Kang et al. [65]. The resulting $C_{1}$ coefficient is very sensitive to both the spatial distribution of the kinks and the assumed character angles of the kinks, with the screw dislocation being particularly sensitive. We will report on more details of this type of analyses in the future, but these studies are generally consistent with the differences between smooth continuum elasticity and our atomistic results. Moreover, for a self-similar geometry, i.e. fixed ratio of maximum bow-out $h$ to length $L$, a longer length simply has more kinks or, equivalently, the kink density depends only on $h / L$ and not $L$, and hence kink-kink interactions would not diminish in importance for larger $L$. The difference between atomistic and elasticity $\ln (L / b)$ scaling is the second main result of this paper.

The differences between elasticity and atomistic results can be further revealed independently of the dislocation line tension computed here. Specifically, we can compare the bow-out configurations as predicted by elasticity and as measured in atomistic simulations after fitting the adjustable elasticity parameters $\left(\mathrm{r}_{0}\right.$ and $\left.\mathrm{r}_{c} / \mathrm{E}_{\text {core }}\right)$ to just one of the atomistic results. We have replicated the atomistic $\mathrm{L}=25 \mathrm{~nm}$ edge bowout using both the Non-Singular theory as implemented within ParaDis and our own simple DD code which truncates neighboring dislocation segment interactions within a cut-off radius $\mathrm{r}_{0}$. Specifically, we chose the core parameters in ParaDiS as $r_{c} / b=2.718$ and $E_{\text {core }}=\frac{2.03 \mu}{4 \pi}$ and within our own code as $\mathrm{r}_{0} / b=1$ and $E_{\text {core }}=\frac{4.83 \mu}{4 \pi}$ to reproduce the edge bow-out configuration for $\mathrm{L}=25 \mathrm{~nm}$ at the effective applied shear stress $\tau_{\text {app } \text { eff }}=46$ MPa. The agreement in this fitted case is shown in figure 6a. Holding all adjustable elasticity parameters fixed, we then compare elasticity to the full atomistic simulations for edge and screw bow-outs at different lengths and/or applied stresses, as shown in figure $6 \mathrm{~b}, \mathrm{c}, \mathrm{d}$. In each case, the applied shear stress for each elastic simulation was selected to match the effective applied shear stress $\left(\tau_{a p p, e f f}\right)$ computed via eq. 11 . The edge dislocation with $\mathrm{L}=50 \mathrm{~nm}$ shows excellent agreement using both elasticity and the Non-Singular theory. This is consistent with independent agreement in the line tension coefficient $C_{1}$. In contrast, there is significant deviation between all three bow-outs for the two screw $(\mathrm{L}=25 \mathrm{~nm}$ and $\mathrm{L}=50 \mathrm{~nm})$ dislocation comparisons. The discrepancy in the $\mathrm{L}=25 \mathrm{~nm}$ screw case is due to the use of a single set of cutoff parameters $\left(\mathrm{r}_{0}\right.$ and 
This is an author-created, un-copyedited version of an article accepted for publication/published in Modelling and Simulation in Materials Science and Engineering. IOP Publishing Ltd is not responsible for any errors or omissions in this version of the manuscript or any version derived from it. The Version of Record is available online at http://dx.doi.org/10.1088/0965-0393/23/8/085008. Citation details: Leyson, G. P. M.; Curtin, W. A. Model. Simul. Mater. Sci. Eng. 2016, 24 (6), 65005.

$\left.r_{c} / E_{\text {core }}\right)$ obtained for the edge dislocation which, while convenient, is an assumption that has no physical basis. That is, there is no rational to suggest that $\mathrm{r}_{0}^{\text {edge }}=r_{0}^{\text {screw }}$ or $r_{c}^{\text {edge }}=r_{c}^{\text {screw }}$ and $E_{\text {core }}^{\text {edge }}=E_{\text {core }}^{\text {screw }}$, as noted in Hirth and Lothe [22]. For the $\mathrm{L}=50 \mathrm{~nm}$ screw case, additional error arises due to the discrepancy in $C_{1}$, since $C_{1}^{\text {aniso }} / C_{1}^{\text {atom }} \approx 2$. These direct comparisons of configurations are thus entirely consistent with the differences in line tension. The inability to match atomistic configurations using standard discretedislocation modeling techniques, even after fitting parameters to match one atomistic case, is the third main result of this paper.

Finally, we address the range of line tension $(\Gamma)$ values published previously in the literature. The combination of processing and post-processing schemes employed here in computing $\Gamma$ are far beyond those in any of the earlier attempts $[2,42-46,48,49]$. First, spurious effects are caused by finite simulation volumes because the image stresses on a bowing-out dislocation scale linearly with the amount of bow-out [50], leading to error in $\tau_{a p p}$ and thus $\Gamma$. Shenoy et al. [44] estimated the image stress and corrected their calculated line tension but the final results remain qualitative. The use of large stress increments $\left(\Delta \tau_{a p p}\right)$ is computationally efficient but the existence of a Peierls energy barrier in conjunction with inertial effects [42] of the gliding dislocation creates a continuous range of metastable equilibrium configurations; we have observed this explicitly in our simulations and have used sufficiently small load increments to avoid such issues. Here, we find that it is essential to introduce an effective Peierls stress for curved dislocations. Thus, neglecting the Peierls stress (e.g. [44]) or assuming it is equal to the value for the straight dislocation (e.g. [51]) both introduce significant errors. For the Al edge, the effective Peierls stress is small and so in this particular case it can be safely neglected. The line tension is a configurational force acting on a smooth, continuous bow-out (e.g. [22]) and is not the line energy, as used in refs. [2,42]. It is also important to account for ellipticity, which has been neglected in all atomic scale studies of dislocations. Finally, the concept of line tension, as applied to a smooth, continuous bow-out, is inapplicable for very small bow-outs (kink density $\rho<\sim 0.05$; Figure 4), and results in this domain are not consistent [48].

\section{Summary}

We have reported accurate, atomically-resolved measurements of the dislocation line tension for the periodic bow-out of both initially edge and screw dislocations, using two distinct interatomic potentials. This has been achieved with the use of a unique 3D multiscale method that eliminates all spurious image forces [52] and with the development of new analysis methods. We have also introduced, out of necessity, the concept of an effective Peierls stress applicable to curved, bow-out dislocation configurations to accurately account for the measured forward and reverse motion of curved dislocations. We have found that, up to lengths of $L=50 \mathrm{~nm}$, the $\ln (L / b)$ dependence of the dislocation line tension for the screw is much weaker than that predicted by elasticity theory, and weakly dependent on the interatomic potential. With 
This is an author-created, un-copyedited version of an article accepted for publication/published in Modelling and Simulation in Materials Science and Engineering. IOP Publishing Ltd is not responsible for any errors or omissions in this version of the manuscript or any version derived from it. The Version of Record is available online at http://dx.doi.org/10.1088/0965-0393/23/8/085008. Citation details: Leyson, G. P. M.; Curtin, W. A. Model. Simul. Mater. Sci. Eng. 2016, 24 (6), 65005.

all of these results and direct comparisons between atomistic and continuum simulations, we have shown that there are inherent limits to the ability of continuum-level dislocation models to quantitatively reproduce atomistic simulations of dislocation behavior. The present results can be used (i) to best-fit continuum dislocation parameters in existing models, (ii) to provide reference configurations for future continuum methods to match, and (iii) to provide quantitative numerical values of the line tension for use in analytic theories of plasticity phenomena. 
This is an author-created, un-copyedited version of an article accepted for publication/published in Modelling and Simulation in Materials Science and Engineering. IOP Publishing Ltd is not responsible for any errors or omissions in this version of the manuscript or any version derived from it. The Version of Record is available online at http://dx.doi.org/10.1088/0965-0393/23/8/085008. Citation details: Leyson, G. P. M.; Curtin, W. A. Model. Simul. Mater. Sci. Eng. 2016, 24 (6), 65005.

\section{Appendix A.}

The line tension $\Gamma^{e l}$ of a dislocation within anisotropic elasticity depends on both the character angle $\phi$ of the nominally straight dislocation and a second orientation vector with respect to the cardinal crystal axes. For convenience we have chosen the unit vectors $\mathbf{n}=[111] / \sqrt{3}$ to represent the glide plane normal, and $\mathbf{t}$ to represent the dislocation line direction, so $\mathbf{b} \cdot \mathbf{t}=|\mathbf{b}| \cos (\phi)$. We may then define a third, mutually perpendicular vector as

$$
\mathbf{m}=\mathbf{n} \times \mathbf{t}
$$

Following along the lines of Barnett and coworkers $[54,55]$ and more recently Aubry et al [53] the dislocation line energy (per unit length) scales with $\ln (L)$ as

$$
W_{\text {self }}(\mathbf{t}(\phi), \mathbf{n})=\mathbf{b} \cdot \mathbf{B}(\mathbf{t}, \mathbf{n}) \cdot \mathbf{b} \ln (L),
$$

where the second-order tensor $\mathbf{B}$ is defined as

$$
\begin{aligned}
\mathbf{B}(\mathbf{t}, \mathbf{n}) & =\frac{1}{8 \pi}(\mathbf{m} \otimes \mathbf{m}+\mathbf{n} \otimes \mathbf{n}): \mathbf{C} \\
& -\frac{1}{4 \pi^{2}} \int_{0}^{\pi}(\mathbf{M} \cdot \mathbf{C} \cdot \mathbf{N}) \cdot(\mathbf{N} \cdot \mathbf{C} \cdot \mathbf{N})^{*} \cdot(\mathbf{N} \cdot \mathbf{C} \cdot \mathbf{M}) \mathrm{d} \eta
\end{aligned}
$$

with $\mathbf{C}$ denoting the stiffness tensor, $(\mathbf{N} \cdot \mathbf{C} \cdot \mathbf{N})^{*}$ defined as the inverse of $(\mathbf{N} \cdot \mathbf{C} \cdot \mathbf{N})$ satisfying $(\mathbf{N} \cdot \mathbf{C} \cdot \mathbf{N})^{*} \cdot(\mathbf{N} \cdot \mathbf{C} \cdot \mathbf{N})=\mathbf{I}$ and the vector(s) over which the integral is taken, $\mathbf{M}$ and its derivative $\mathbf{N}$ defined conveniently in terms of a single angle, $\eta$ as

$$
\begin{aligned}
& \mathbf{M}=\mathbf{m} \cos (\eta)+\mathbf{n} \sin (\eta) \\
& \mathbf{N}=-\mathbf{m} \sin (\eta)+\mathbf{n} \cos (\eta)
\end{aligned}
$$

The integral in $\mathbf{B}$, as noted by Aubry et al [53], involves the ratio of a fourth-order polynomial to a sixth-order polynomial and so is analytically insoluble. Any numerical integration technique however, such as Simpson's rule will suffice. Given $W_{\text {self }}(\mathbf{t}(\phi), \mathbf{n})$, the line tension prefactor may be computed numerically as

$$
C_{1}(\phi, \mathbf{n}) \ln (L)=\left(W_{\text {self }}(\mathbf{t}(\phi), \mathbf{n})+\frac{\partial^{2} W_{\text {self }}(\mathbf{t}(\phi), \mathbf{n})}{\partial \phi^{2}}\right)
$$

\section{Acknowledgments}

BAS is grateful for fruitful discussions with DJ Bacon. The authors thank the Fonds National Suisse for support of this work through the project 200021_140506 entitled "Coupled Atomistic/Discrete-Dislocations in 3d (CADD3d)", and also gratefully acknowledge additional support during the final stages of this work from the European Research Council Advanced Grant "Predictive Computational Metallurgy", ERC Grant agreement No. 339081 - PreCoMet. 
This is an author-created, un-copyedited version of an article accepted for publication/published in Modelling and Simulation in Materials Science and Engineering. IOP Publishing Ltd is not responsible for any errors or omissions in this version of the manuscript or any version derived from it. The Version of Record is available online at http://dx.doi.org/10.1088/0965-0393/23/8/085008. Citation details: Leyson, G. P. M.; Curtin, W. A. Model. Simul. Mater. Sci. Eng. 2016, 24 (6), 65005.

Robust Atomistic Calculation of Dislocation Line Tension

\section{References}

[1] Terentyev D, Bacon D J and Osetsky Y N 2008 Interaction of an edge dislocation with voids in $\alpha$-iron modelled with different interatomic potentials J. Phys. Condens. Mat. 20445007

[2] Proville L and Patinet S 2010 Atomic-scale models for hardening in fcc solid solutions Phys. Rev. B 82054115

[3] Tang Y and El-Awady A 2012 Atomistic simulations of the interactions of hydrogen with dislocations in fcc metals Phys. Rev B 86174102

[4] Osetsky Y N, Bacon D J and Mohles V 2003 Atomic modelling of strengthening mecanisms due to voids and copper precipitates in $\alpha$-iron Phil. Mag. 833623 - 41

[5] Tapasa K, Osetsky Y N and Bacon D J 2007 Computer simulation of interaction of an edge dislocation with a carbon interstitial in $\alpha$-iron and effects on glide Acta Mater. 55 93-104

[6] Rodney D and Martin G 1999 Dislocation pinning by glissile interstitial loops in a nickel crystal: A molecular-dynamics study Phys. Rev. B 61 8714-25

[7] Rodney D 2004 Molecular dynamics simulation of screw dislocations interacting with interstitital frank loops in a model fcc crystal Acta Mater. 52 607-14

[8] Osetsky Y N and Bacon D J 2003 An atomic-level model for studying the dynamics of edge dislocations in metals Modelling Simul. Mater. Sci. Eng. 11 427-46

[9] Osetsky Y N and Bacon D J 2005 Comparison of void strengthening in fcc and bcc metals: Largescale atomic-level modelling Mater. Sci. Eng. A 400-401 374-377

[10] Dong Y and Curtin W A 2012 Thermally activated plastic flow in the presence of multiple obstacle types Modelling Simul. Mater. Sci. Eng. 20075006

[11] Xu Z and Picu R C 2007 Thermally activated motion of dislocations in fields of obstacles: The effect of obstacle distribution Phys. Rev. B $\mathbf{7 6} 094112$

[12] Hasnaoui A, Derlet P M and Van Swygenhoven H 2004 Interaction between dislocations and grain boundaries under an indenter - a molecular dynamics simulation Acta Mater. 522251 - 8

[13] Farkas D, Froseth A G and Van Swygenhoven H 2006 Grain boundary migration during room temperature deformation of nanocrystalline Ni Scripta Mater. 55695 - 8

[14] Velasco M, Van Swygenhoven H and Brandl C 2011 Coupled grain boundary motion in a nanocrystalline grain boundary network Scripta Mater. $65151-4$

[15] Van Swygenhoven H, Derlet P M and Hasnaoui A 2003 Atomistic modelling of strength of nanocrystalline metals Adv. Eng. Mater. $5345-50$

[16] Froseth A G, Derlet P M and Van Swygenhoven H 2005 Twinning in nanocrystalline fcc metals Adv. Eng. Mater. $716-20$

[17] Bulatov V V, Hsiung L L, Tang M, Arsenlis A, Bartelt M C, Cai W, Florando J N, Hiratani M, Rhee M, Hommes G, Pierce T G and Rubia T D 2006 Dislocation multi-junctions and strain hardening Nat. Lett. $4401174-8$

[18] Greer J R 2013 Nanotwinned metals: It's all about imperfections Nat. Mater. 12689 - 90

[19] Weinberger C R and Cai W 2008 Surface-controlled dislocation multiplication in metal micropillars P. Natl. Acad. Sci. $10514304-7$

[20] Olmsted D L, Hector Jr. L G and Curtin W A 2006 Molecular dynamics study of solute strengthening in $\mathrm{Al} / \mathrm{Mg}$ alloys J. Mech. Phys. Solids $\mathbf{5 4} 1763-88$

[21] Leyson G P M, Curtin W A, Hector Jr. L G and Woodward C F 2010 Quantitative prediction of solute strengthening in aluminum alloys Nat. Mater. $9750-5$

[22] Hirth J P and Lothe J 1982 Theory of Dislocations Wiley

[23] DeWit G and Koehler J S 1959 Interactions of Dislocations with an Applied Stress in Anisotropic Crystals Physical Review 1161113 - 8

[24] Foreman A J E 1967 The Bowing of a Dislocation Segment Philosophical Magazine 151011 - 11

[25] Bacon D J, Kocks U F and Scattergood R O The effect of dislocation self-interaction on the orowan stress Philosophical Magazine 281241 - 22

[26] Li P, Picu R C and Weiss J 2010 Dynamics below the depinning transition of interacting 
This is an author-created, un-copyedited version of an article accepted for publication/published in Modelling and Simulation in Materials Science and Engineering. IOP Publishing Ltd is not responsible for any errors or omissions in this version of the manuscript or any version derived from it. The Version of Record is available online at http://dx.doi.org/10.1088/0965-0393/23/8/085008. Citation details: Leyson, G. P. M.; Curtin, W. A. Model. Simul. Mater. Sci. Eng. 2016, 24 (6), 65005.

dislocations moving over fields of obstacles Phys. Rev. E $\mathbf{8 2} 022107$

[27] Rodney D and Phillips R 1999 Structure and strength of dislocation junctions: An atomic level analysis Phys. Rev. Lett. $821704-7$

[28] Nogaret T and Rodney D 2006 Finite-size effects in dislocation glide through random arrays of obstacles: Line tension simulations Phys. Rev. B $\mathbf{7 4} 134110$

[29] Dong Y, Nogaret T and Curtin W A 2010 Scaling of dislocation strengthening by multiple obstacle types Metall. Mater. Trans. A 41A $1954-60$

[30] Mott N F and Nabarro F R N 1948 Report on Strength of Solids Physical Society London

[31] Cottrell A H 1953 Dislocations and Plastic Flow in Crystals Clarendon Press Oxford

[32] Friedel K 1964 Dislocations Addison-Wesly Publishing Company

[33] Madec R, Devincre B, Kubin L, Hoc T and Rodney D 2003 The role of collinear interaction in dislocation-induced hardening Science $3011879-82$

[34] Dupuy L and Fivel M C 2002 A study of dislocation junctions in fcc metals by an orientation dependent line tension model Acta Mater. 504873 - 85

[35] Wickham L K, Schwarz K W and Stölken J S 1999 Rules for forest interactions between dislocations Phys. Rev. Lett. $834574-77$

[36] Needleman A and Van Der Giessen E 1995 Discrete dislocation plasticity: A simple planar model Modelling Simul. Mater. Sci. Eng. $3689-735$

[37] Arsenlis A, Cai W, Tang M, Rhee M, Oppelstrup T, Hommes G, Pierce T G and Bulatov V V 2007 Enabling strain hardening simulations with dislocation dynamics Modelling Simul. Mater. Sci. Eng. 15553 - 95

[38] Weygand D, Friedman L H, Van Der Giessen E and Needleman A 2002 Aspects of boundary-value problem solutions with three dimensional dislocation dynamics Modelling Simul. Mater. Sci. Eng. $10437-68$

[39] Khraishi T A, Zbib H M and Rubia T D 2001 The treatment of traction-free boundary condition in three-dimensional dislocation dynamics using generalized image stress analysis Mater. Sci. Eng. A $309283-7$

[40] Devincre B and Kubin L P 1997 Mesoscopic simulations of dislocations and plasticity Mater. Sci. Eng. A $2348-14$

[41] Plimpton S 1995 Fast parallel algorithms for short-range molecular-dynamics J. Comp. Phys. 117 $1-19$

[42] Cheng Y, Bitzek E, Weygand D and Gumbsch P 2010 Atomistic simulation of dislocation-void interactions under cyclic loading Modelling Simul. Mater. Sci. Eng. 18025006

[43] Brailsford A D 1965 Effective line tension of a dislocation Phys. Rev. 1391813 - 17

[44] Shenoy V B and Phillips R 1997 Finite-sized atomistic simulations of screw dislocations Phil. Mag. A 76367

[45] Proville L, Ventelon L and Rodney D 2013 Prediction of the kink-pair formation enthalpy on screw dislocations in $\alpha$-iron by a line tension model parameterized on empirical potentials and first-principles calculations Phys. Rev. B $\mathbf{8 7} 144106$

[46] Rodney D and Proville L 2009 Stress-dependent peierls potential: Influence on kink-pair activation Phys. Rev. B 79094108

[47] Zhou X, Ward D K, Wong B M, Doty P F and Zimmerman J A 2012 Molecular dynamics studies of dislocations in cdte crystals from a new bond order potential J. Phys. Chem. $11617563-71$

[48] Patinet S and Proville L 2011 Dislocation pinning by substitutional impurities in an atomic-scale model for $\mathrm{Al}(\mathrm{Mg})$ solid solutions Phil. Mag. 911581 - 1606

[49] Bacon D J 2009 Dislocation-obstacle interactions at the atomic level Dislocations in Solids 151 90

[50] Szajewski B A and Curtin W A 2015 Analysis of spurious image forces in atomistic simulations of dislocations Modelling Simul. Mater. Sci. Eng. 23025008

[51] Dong Y 2013 Coupled Dislocation/Dislocation and Solute Strengthening Mechanisms in Metal Alloys PhD thesis, Brown University 
This is an author-created, un-copyedited version of an article accepted for publication/published in Modelling and Simulation in Materials Science and Engineering. IOP Publishing Ltd is not responsible for any errors or omissions in this version of the manuscript or any version derived from it. The Version of Record is available online at http://dx.doi.org/10.1088/0965-0393/23/8/085008. Citation details: Leyson, G. P. M.; Curtin, W. A. Model. Simul. Mater. Sci. Eng. 2016, 24 (6), 65005.

[52] Pavia F and Curtin W A 2015 Parallel Algorithm for Multiscale Atomistic/Continuum Simulations using LAMMPS Modelling Simul. Mater. Sci. Eng. 23055002

[53] Aubry S, Fitzgerald S P and Arsenlis A 2014 Methods to compute dislocation line tension energy and force in anisotropic elasticity Modelling Simul. Mater. Sci. Eng. 22015001

[54] Bacon D J, Barnett D M and Scattergood R O 1979 Anisotropic continuum theory of lattice defects Prog. Mater. Sci. $2351-262$

[55] Barnett D M, Asaro R J, Gavazza S D, Bacon D J and Scattergood R O 1972 The effects of elastic anisotropy on dislocation line tension in metalsF J. Phys. F: Metal Phys. $2854-64$

[56] Cai W, Athanasios A, Weinberger C R and Bulatov V V 2006 A non-singular continuum theory of dislocations J. Mech. Phys. Solids $54561-87$

[57] Fitzgerald S P and Aubry S 2010 Self-force on dislocation segments in anisotropic crystals J. Phys. Condens. Matter 22295403

[58] Indenbom V L and Lothe J 1992 Elastic Strain Fields and Dislocation Mobility North-Holland (March 1992)

[59] Ercolessi F and Adams J B 1994 Interatomic potentials from first-principles calculations: The force-matching method Europhys. Lett. $26583-8$

[60] Arsenlis A, Cai W, Tang M, Rhee M, Oppelstrup T, Hommes G, Pierce T G and Bulatov V V 2007 Enabling Strain Hardening Simulations with Dislocation Dynamics Modelling Simul. Mater. Sci. Eng $15553-42$

[61] Mishin Y, Farkas D, Mehl M J and Papconstantopoulos D A 1999 Interatomic potentials for monoatomic metals from experimental data and Ab Initio calculations Phys. Rev. B 5933393 $-3407$

[62] Li J 2003 Atomeye: an efficient atomistic configuration viewer Modelling Simul. Mater. Sci. Eng. $11173-7$

[63] Cash W D and Cai W 2011 Dislocation Contribution to Acoustic Nonlinearity: The Effect of Orientation-Dependent Line Energy Journal of Applied Physics 10901415

[64] Peach M, JS Koehler 1950 The Forces Exerted on Dislocations and the Stress Fields Produced by Them Physical Review 80436

[65] Kang K and Bulatov V V and Cai W 2012 Singular orientations and faceted motion of dislocations in body-centered cubic crystals Proceedings of the National Academy of Sciences of the United States of America 10915174 